NASA $\angle$ FM-113011

\title{
HEALTH MONITORING SYSTEM FOR COMPOSITE STRUCTURES
}

\author{
S. S. Tang and P. C. Riccardella \\ Structural Integrity Associates, Inc. \\ San Jose, California \\ A. N. Mucciaradi \\ Infometrics \\ Silver Spring, Maryland
}

\author{
$1-7-7<$ \\ R. J. Andrews \\ University of Dayton Research Institute \\ Dayton, Ohio \\ J. E. Grady \\ NASA, Lewis Research Center \\ Cleveland, Ohio
}

\begin{abstract}
An automated system was developed to monitor the health status of composites. It uses the vibration characteristics of composites to identify a component's damage condition. The vibration responses are characterized by a set of signal features defined in the time, frequency and spatial domains. The identification of these changes in the vibration characteristics corresponding to different health conditions was performed using pattern recognition principles. This allows efficient data reduction and interpretation of vast amounts of information. Test components were manufactured from isogrid panels to evaluate performance of the monitoring system. The components were damaged by impact to simulate different health conditions. Free vibration response was induced by a tap test on the test components. The monitoring system was trained using these free vibration responses to identify three different health conditions. They are undamaged vs. damaged, damage location and damage zone size. High reliability in identifying the correct component health condition was achieved by the monitoring system.
\end{abstract}

\section{Monitoring Principles}

The damage monitoring of composite using pattern recognition principles has been shown to be feasible ${ }^{1}$ with a limited amount of data from a composite cantilever beam. The changes in structural vibration can be associated with the damage in a monitored structure. ${ }^{2-6}$ These changes can be efficiently interpreted through the use of pattern recognition method. The application of pattern recognition method, $1,7,8$ requires prior knowledge in the correct classification of an output class using available input information of a monitored structure. The knowledge can be acquired through a training process. This process uses a database of relevant input information that corresponds to a defined monitored health condition of the structure. To obtain the necessary information, the input data can be acquired from a network of suitable sensors. This input information can be described as a feature vector. The features are defined according to a specific application. The feature information is used in the training of a monitoring system to obtain an optimum feature set for a specific classification of output This optimum feature set is used by the classifiers to perform the output classification. The commonly used classifiers in pattem recognition are Nearest Neighbor Criteria (NNC), Gaussian and Fisher. ${ }^{8}$

\section{Composite Structure Health Monitoring System}

A health monitoring system for composite structures, Figure 1, was developed on a microprocessor computer to implement the above principles in the classification of structural component's health conditions. A schematic of the monitoring system is presented in Figure 2. The system consists of a 16 channel signal conditioner, a post-amplifier with noise filler, and an analog-to-digital (AD) card plugged into a rack mounted $486 / 33 \mathrm{MHz}$ personal computer. The AD card is capable of digitizing data up to $150 \mathrm{KHz}$ for one channel. An integrated software was developed for the system, Figure 3. This software is menu driven It's capabilities include data acquisition, signal processing, feature extraction, classification, and file management. On screen calibration procedures are also provided. Classification results on the component health condition are provided at the end of data acquisition. Data can be saved in files for further training, evaluation or archive.

\section{Test Components}

The test components were manufactured from isogrid panels. The panels were fabricated using $\mathbb{M}-7$ fiber and $977-2$ epoxy. The panels were $58 \mathrm{~cm}\left(23^{\prime \prime}\right)$ by $50.5 \mathrm{~cm}$ (20"). They consisted of a twelve ply graphite/epoxy skin with ply thickness of approximately $2 \mathrm{~mm}\left(0.079^{\prime \prime}\right)$ and stiffener ribs $1.5 \mathrm{~cm}\left(0.6^{\prime \prime}\right)$ high. Fiber direction was unidirectional along the ribs and $[+-60,0,0,-+60]_{S}$ in the skin, as shown Figure 4 . The isogrid panel material

1

Copyright 01996 by the American Institute of Aeronautics and Astronautics Inc. All rights reserved. 
properties are presented in Table 1. Four test components were cut from each panel. The test component dimensions were $17.8 \mathrm{~cm}\left(7^{\prime \prime}\right)$ by $22.9 \mathrm{~cm}\left(9^{\circ}\right)$, Figure 5. A total of 28 components were made from seven isogrid panels.

The test components were first inspected by ultrasonic $\mathrm{C}$-scan to document the undamaged status. Then damages were introduced on the test components by impact. Among the 28 components, 20 were selected to be damaged by impact. Impacts were set at different energy levels to obtain a range of damage sizes. Damages were induced in the test components at two locations; center and off-center, Figure 6 . The test components were again inspected by ultrasonic $\mathrm{C}$-scan to document the actual damage locations and sizer. Inspection results found damage sizes measured from $4 \mathrm{~mm}$ to $33.5 \mathrm{~mm}$ in diameter. Table 2 summarizes the measurement results on the damage sizes and locations. Figure 6 presents a comparison of the C-scans before and after the impact damage of the same test component.

The components were instrumented with 16 strain gages mounted in a $4 \times 4$ equi-distance grid on the components, as shown in Figure 5. The free vibration was initiated by tapping the component hanging from a metal stand with a bungy cord. Eight tap tests were performed on each component, before and after they were damaged by impact. Some typical waveforms of free vibration from selected channels are presented in Figure 7. Corresponding frequency spectra are presented in Figure 8 . These vibration results formed a database for the training and validation of the composite structure health monitoring system.

\section{Eeature Definition and Extraction}

For the sixteen channels of strain gage, a total of 2,424 signal features were defined. A maximum of 149 features can be utilized for each channel. These signal features describe the signal amplitude, range, variance and cumulative distribution in the time domain In the frequency domain, frequency bins are used to define the highest peak frequency, bandwidth, number of peaks, and energy in each bin. Amplitude ratios of the highest peaks among different bins are also defined. A maximum of 20 frequency bins can be used in each channel. The spatial domain features are defined using all 16 sensors. The spatial features use the amplitude at each spatial frequency and the fall rates among the spatial frequencies.

\section{Svstem Performance Evaluation}

The monitoring system was trained and evaluated for performance on the following component health status:

(a) health condition: undamaged or damaged,

(b) damage location: center or off center, and

(c) damage size: small or large.

The free vibration response database consisted of 384 sets of 16 waveforms acquired from 28 undamaged components and 20 damage components. Table 3 presents a test matrix. The test matrix identified the number of components assigned to each class of health condition. It also identified the number of components to be used in the system training. The damage locations were divided into two classes; center and off-center. Damage sizes were divided into two classes; small $\left(<1.65 \mathrm{~cm} / 0.65^{\prime \prime}\right)$ and large $\left(>1.65 \mathrm{~cm} / 0.65^{\prime \prime}\right)$. Five tap tests were randomly selected from each component in this set to be used in the training of the monitoring system.

Two approaches were used in the training. They were identified as:

(a) Classification without feature normalization

(b) Classification with feature normalization.

In feature normalization, all signal features from each component, undamaged or damage, were normalized by the corresponding feature means calculated from the eight tap tests of the corresponding undamaged component The feature normalization was needed in order to maintain a robust performance which could be affected because of material property variations from component to component. Figure 9 presents a comparison of features from undamaged and damage conditions without feature normalization. The first part of the curve is the data from the undamaged component, (IDs 11-74). The second part of the curve is the data from the damaged components, (IDs 42-31). The damage size was also plotted in the figure. The undamaged components were represented with zero damage size. It shows a wide variation of feature values within each class. There is no distinction in the feature values between the undamaged and damaged components. A similar comparison is presented in Figure 10 using feature normalization. With feature normalization, there is a distinct difference in the feature between the undamaged and damage conditions of the composite components. The damage components have a higher feature magnitude and larger feature variance.

The results on monitoring system training for the classification of three health conditions are summarized in 
Table 4. The training errors in identifying the correct health status using the two approaches were from about $1 \%$ to $14 \%$. In all cases, no more than four features were selected in each of the optimum feature sets. The majority of the optimum features were peak frequency amplitude ratios among different frequency bins. Peak frequency bandwidth was the next common optimum feature.

After the training, all data was used in the performance evaluation of the monitoring system. The overall performance reliability of the system was compiled based on the number of correct calls on test components' health status. System reliability was evaluated on a "component" basis. The decision on a component basis health status was by the majority vote of the outcome of classification from the eight tap tests performed on each component. This can be the choice for monitoring because the inspection personnel is likely to obtain several tests on the component before a decision is made on the component's health status.

The monitoring system reliability results are summarized in Table 5. Between the two approaches, feature normalization produced consistent and reliable performances in identifying the components' correct health status. With feature normalization, the system had a reliability level of over $90 \%$ in the classification of health status, undamaged or damage. Also, the performance reliability was consistent among all three classifiers and in both training and performance evaluation. Performance reliability was not as consistent in the classification of damage location. The NNC classifier achieved an accuracy of $80 \%$ in making the correct call. In identifying the correct damage size in the composite components, the NNC classifier had a reliability of over $95 \%$. Without feature nomalization, the overall accuracies in classification were not as good and consistent. The highest reliability was about $85 \%$ in identifying the damage location. The NNC classifier consistently had the best performance among the three classifiers, with or without the feature normalization.

To visualize the classification results, the optimum features from each classification were plotted using the principle components method. The principle components are the eignvalues of the optimum feature set. The two largest eignvalues are used as the two principle component axes. The transformation of the optimum feature set was performed using the eignvectors corresponding with the two largest eignvalues. Figures 11 and 12 present the class clustering for each monitored component health condition. Without feature normalization, the overlapping of two classes in the feature space was more prominent. With feature normalization, the class clustering was better defined, as presented in Figures 13 to 15. A higher degree of class overlapping and scatter distribution of features corresponded to a lower reliability in identifying the correct component health status by the monitoring system.

\section{Conclusions}

A health monitoring system for composite structure has been developed. The system is capable of monitoring three different health conditions of a composite structure. These three health conditions are undamaged and damage, damage location, and damage size. Test components were manufactured from composite isogrid panels to evaluate the performances of the monitoring system. A very good overall reliability of the system was achieved in all three monitored health conditions. Of the two approaches in classification of health condition developed for the health monitoring systems, feature normalization produced a better system performance reliability.

\section{References}

1. Tang, S. S., Chen, K I. and Grady, J., "On the Monitoring of Degradation of Composite Materials Using Pattern Recognition Method," 7th Technical Conference, ASM, University Park, PA. Oct 1992.

2. Chamis, C. C., Sinclair, J. H and Lark, R F., "Dynamic Response of Damage Angleplied Fiber Composites," Modern Development in Composite Materials and Structures, Winter Annual Meeting, New York, Dec. 1979.

3. Shen, MH.H and Grady, J.E., "Free Vibration of Delaminated Beams," 32nd Structures, Structural Dynamics, and Material Conference, Paper AAIA-91124-CP, Baltimore, MD. April, 1991.

4. Wolff, $T$. and Richardson, M, "Fault Detection in Structures from Changes in their Modal Parameters," Proceedings of 7 th MAC Conference, Las Vegas, Nevada, Jan. 1989.

5. DiBenedetto, A.T., Gauchel, J. V., Thomas, R. L. and Barlow, J. W., "Nondestructive Determination of Fatigue Crack Damage in Composites Using Vibration Tests," Journal of Materials, MISA, Vol. 7, No. 2, June 1972.

6. Adams, R D., Walton, D., Flitcroft, J. E. and Short, D., "Vibration Testings as a Nondestructive Test Tool for Composite Materials," Composite Reliability, ASTM STP 580, 1975. 
7. Fukunaga, K, "Introduction to Statistical Patter Recognition," 2nd Edition, Academic Press, 1990.

8. Tou, J. T. and Gonzalez, R. C., "Pattern Recognition Principles," Addison-Wesley Publishing Co., Massachusetts, 1974.
Table 1. Material Properties of Isoprid Panel

\begin{tabular}{|c|c|c|}
\hline & SKIN & RIB \\
\hline \hline$E_{\gamma}, \times 10^{6} \mathrm{psi}$ & 8.63 & \\
\hline $\mathrm{E}_{\mathrm{\gamma}}, \times 10^{6} \mathrm{psi}$ & 8.63 & \\
\hline $\mathrm{G}_{\mathrm{\alpha}}, \times 10^{6} \mathrm{psi}$ & 3.30 & \\
\hline $\mathrm{NU}_{\gamma}$ & 0.3 & \\
\hline $\mathrm{E}_{1}, \times 10^{6} \mathrm{psi}$ & & 22.6 \\
\hline $\mathrm{E}_{2}, \times 10^{6} \mathrm{psi}$ & & 1.21 \\
\hline $\mathrm{G}_{12}, \times 10^{6} \mathrm{psi}$ & & 0.85 \\
\hline $\mathrm{NU}_{12}$ & & 0.299 \\
\hline
\end{tabular}

Table 2. Impact Test Results

\begin{tabular}{|c|c|c|c|c|}
\hline \multirow{2}{*}{ Test Sequence } & \multirow{2}{*}{ Damage Location } & \multicolumn{3}{|c|}{ Damage Size } \\
\hline & & Nominal (inch) & Actual (xy) & Average (mm) \\
\hline 1 & Center & 0.5 & $(12.5,12)$ & 12.25 \\
\hline 2 & Center & 1 & $(20,23)$ & 21.5 \\
\hline 3 & Off-Center & 0.5 & $(14,14.5)$ & 14.25 \\
\hline 4 & Off-Center & 1 & $(42,28)$ & 35 \\
\hline 5 & Center & 0.5 & $(9,9)$ & 9 \\
\hline 6 & Center & 1 & $(15.75,17.9)$ & 16.825 \\
\hline 7 & Off-Center & 0.5 & $(15,18)$ & 16.5 \\
\hline 8 & Off-Center & 1 & $(28,24)$ & 26 \\
\hline 9 & Center & 0.5 & $(14,14)$ & 14 \\
\hline 10 & Center & 1 & $(15,16)$ & 15.5 \\
\hline 11 & Off-Center & 0.5 & $(11,11)$ & 11 \\
\hline 12 & Off-Center & 1 & $(36.5,28.4)$ & 32.45 \\
\hline 13 & Center & 0.5 & $(14,13)$ & 13.5 \\
\hline 14 & Center & 1 & $(22,20)$ & 21 \\
\hline 15 & Off-Center & 0.5 & $(15,13)$ & 14 \\
\hline 16 & Off-Center & 1 & $(35,32)$ & 33.5 \\
\hline 17 & Center & 0.5 & $(4,4)$ & 4 \\
\hline 18 & Center & 1 & $(20,21)$ & 20.5 \\
\hline 19 & Off-Center & 0.5 & $(12,11)$ & 11.5 \\
\hline 20 & Off-Center & 1 & $(33.29)$ & 31 \\
\hline
\end{tabular}


Table 3. Test Matrix

\begin{tabular}{|c|c|c|c|c|}
\hline & & \multicolumn{3}{|c|}{ Damage Size } \\
\hline & & None & $\begin{array}{c}<1.65 \mathrm{~cm} \\
\left(0.65^{\prime \prime}\right) \\
\end{array}$ & $\begin{array}{c}>1.65 \mathrm{~cm} \\
\left(0.65^{\prime \prime}\right) \\
\end{array}$ \\
\hline \multicolumn{2}{|c|}{ Undamaged } & $28(20)$ & - & - \\
\hline \multirow{2}{*}{ Damaged } & $\begin{array}{c}\text { Off } \\
\text { Center } \\
\end{array}$ & - & $5(3)$ & $5(4)$ \\
\hline & Center & - & $6(4)$ & $4(3)$ \\
\hline
\end{tabular}

Note: () identifies number of data used in health monitoring system training

Table 4. Summary of the Health Monitoring SystemTraining Results

\begin{tabular}{|c|c|c|c|c||}
\cline { 2 - 5 } \multicolumn{1}{c|}{} & \multicolumn{2}{c|}{ Without Normalization } & \multicolumn{2}{c|}{ With Normalization } \\
\hline Health Status & $\begin{array}{c}\text { Optimum } \\
\text { Feature }\end{array}$ & Training Error & $\begin{array}{c}\text { Optimum } \\
\text { Feature }\end{array}$ & Training Error \\
\hline Damaged and Undamaged & 4 & $12.9 \%$ & 3 & $5.3 \%$ \\
\hline Location & 4 & $1.4 \%$ & 3 & $7.1 \%$ \\
\hline Size & 1 & $11.4 \%$ & 4 & $1.4 \%$ \\
\hline
\end{tabular}

Table 5. Summary of Performance Reliability on the Component Basis by the Health Monitoring System

\begin{tabular}{|c||c|c|c|c|c|c|c|c|}
\hline \multicolumn{1}{|c|}{} & \multicolumn{4}{c|}{ Without Normalization } & \multicolumn{4}{c|}{ With Normalization } \\
\hline \multicolumn{1}{|c|}{ Training } & NNC & Gaussian & Fisher & Training & NNC & Gaussian & Fisher \\
\hline Undamaged Damaged & $88 \%$ & $77 \%$ & $60 \%$ & $63 \%$ & $95 \%$ & $96 \%$ & $96 \%$ & $90 \%$ \\
\hline Location & $98 \%$ & $85 \%$ & $80 \%$ & $80 \%$ & $93 \%$ & $80 \%$ & $65 \%$ & $70 \%$ \\
\hline Size & $89 \%$ & $70 \%$ & $60 \%$ & $60 \%$ & $98 \%$ & $95 \%$ & $80 \%$ & $85 \%$ \\
\hline
\end{tabular}




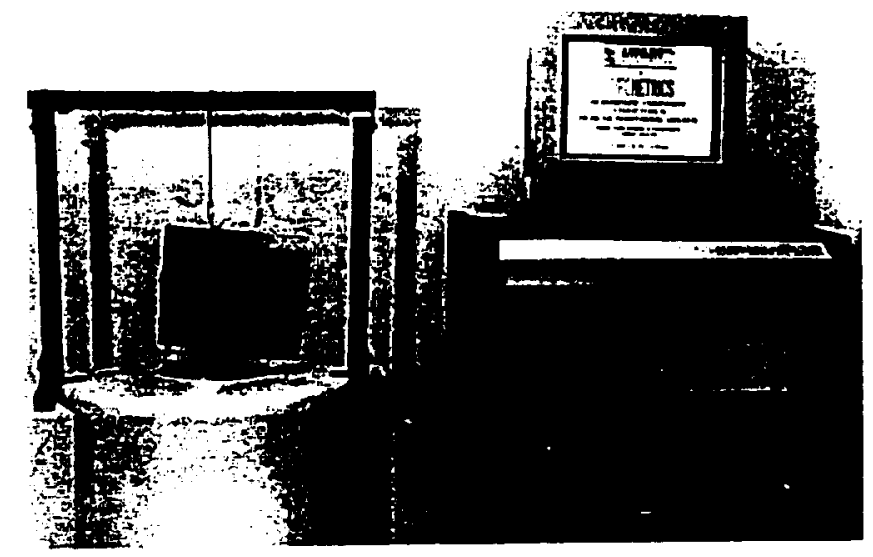

Figure 1. Prototype Health Monitoring System

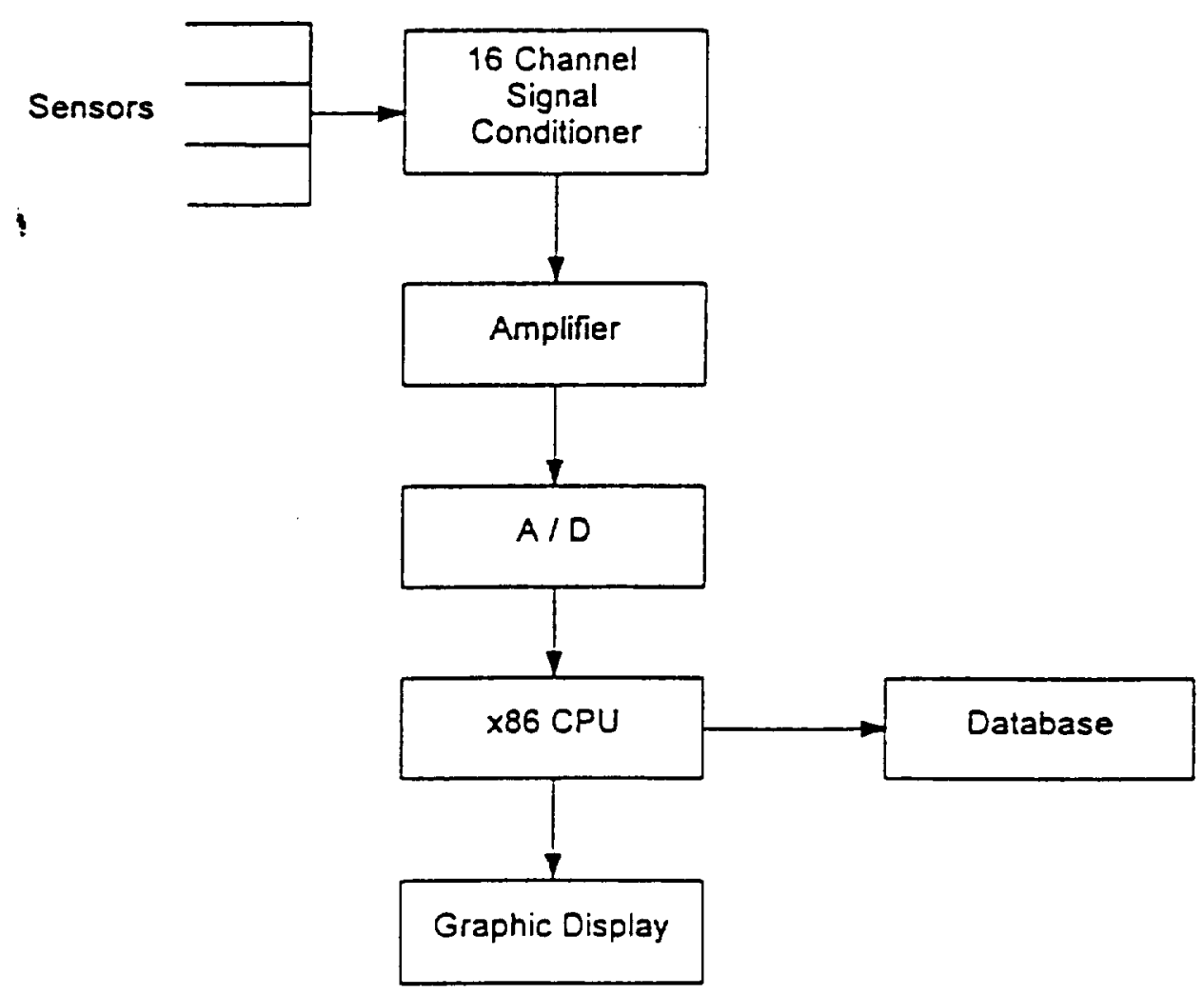

Figure 2. Monitoring System Schematic 


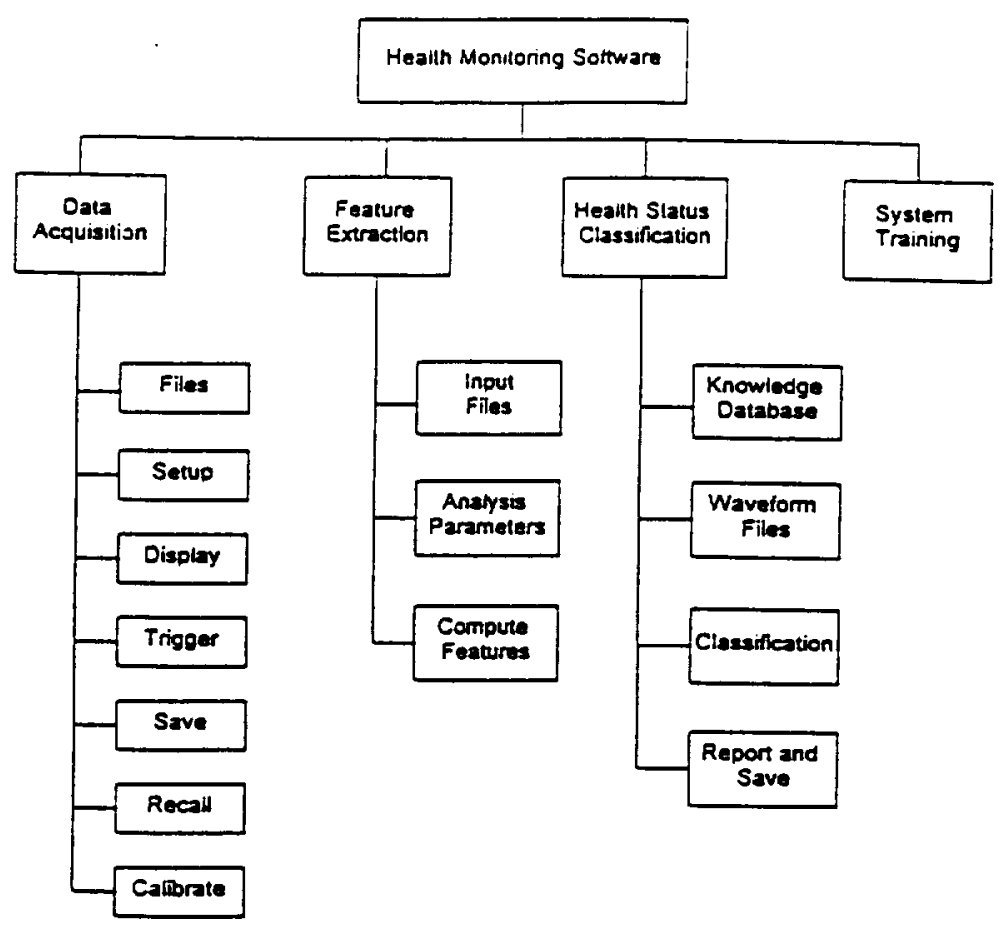

Figure 3. Software Architecture Schematic for Health Monitoring System

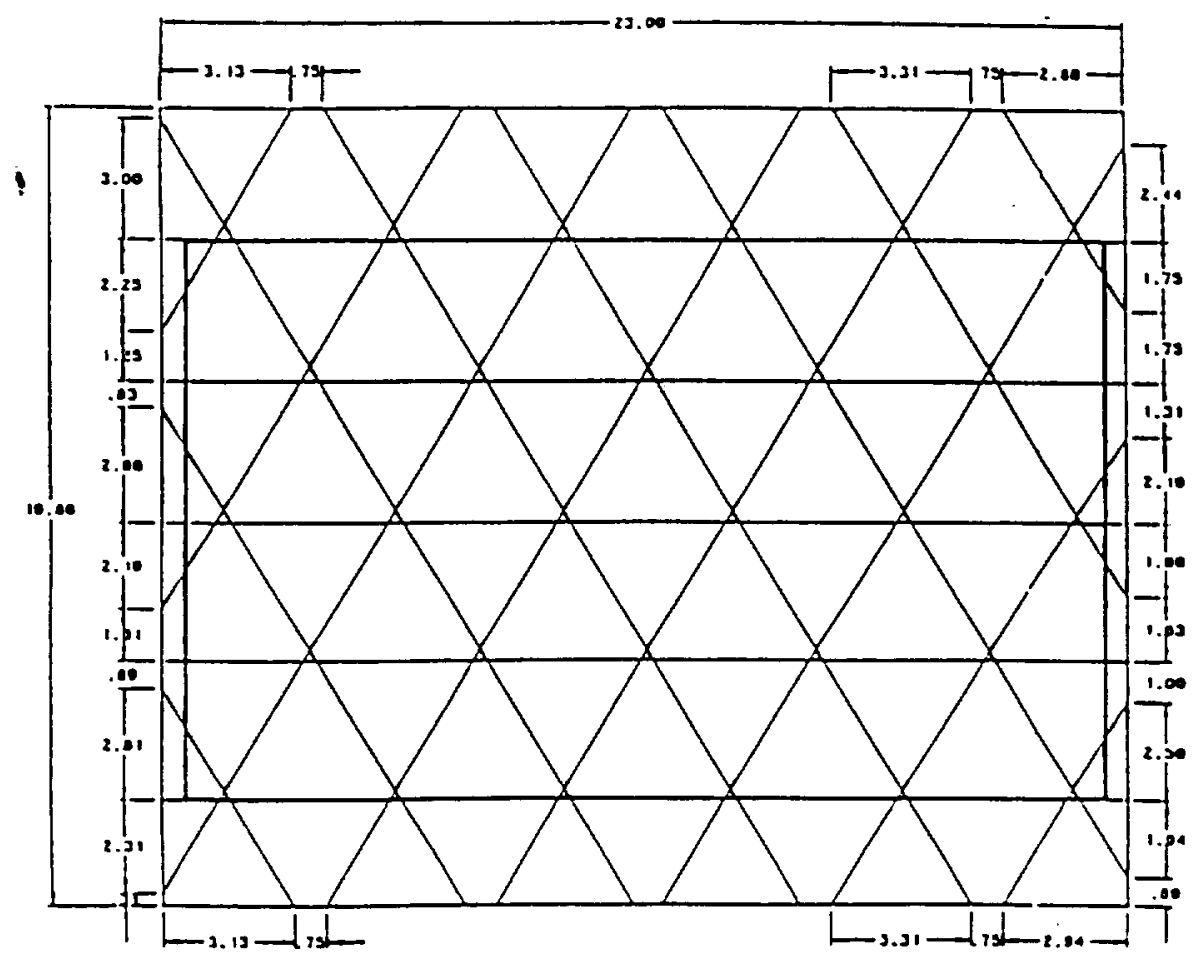

Figure 4. Diagram Showing Rib Pattern on Isogrid Panel 


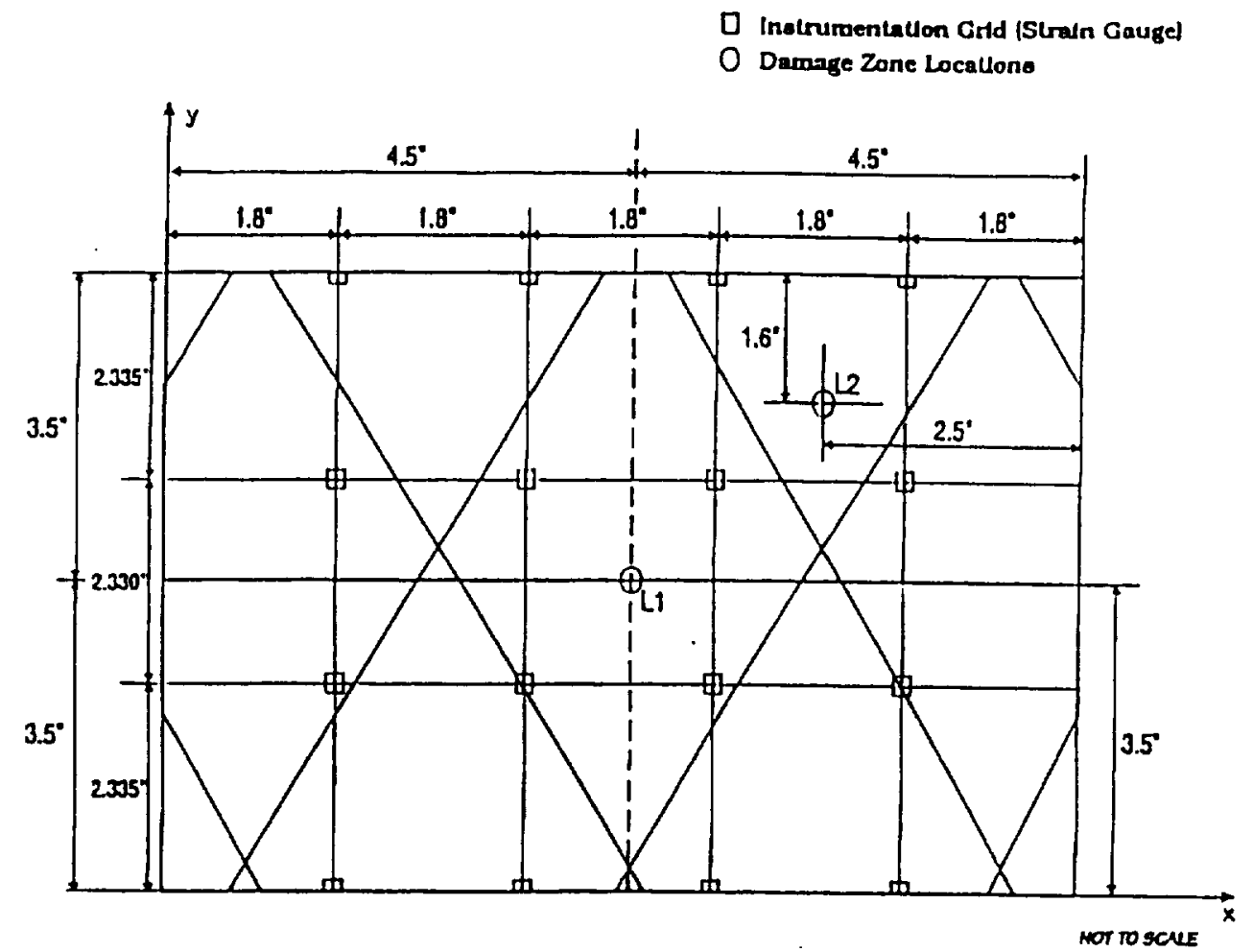

Figure 5. Sketch of Damaged Zone Locations

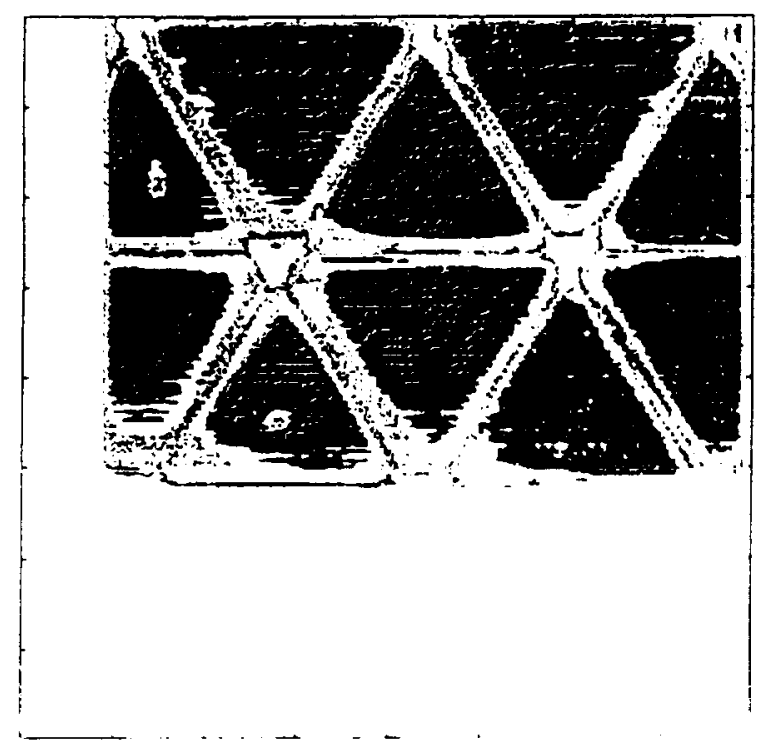

(a) before impact

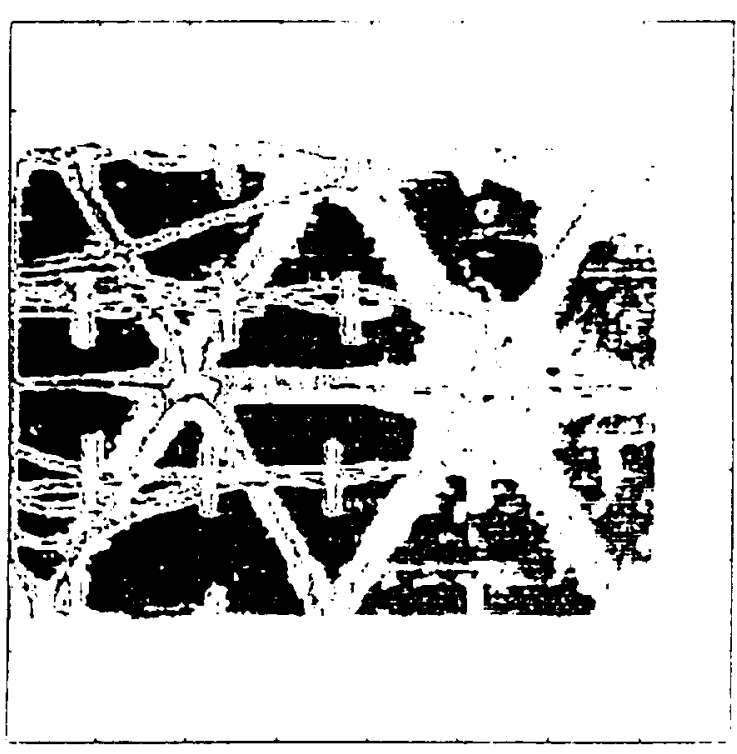

(b) impact at off center

Figure 6. Typical C-Scan Image of Impact Damaged Component 

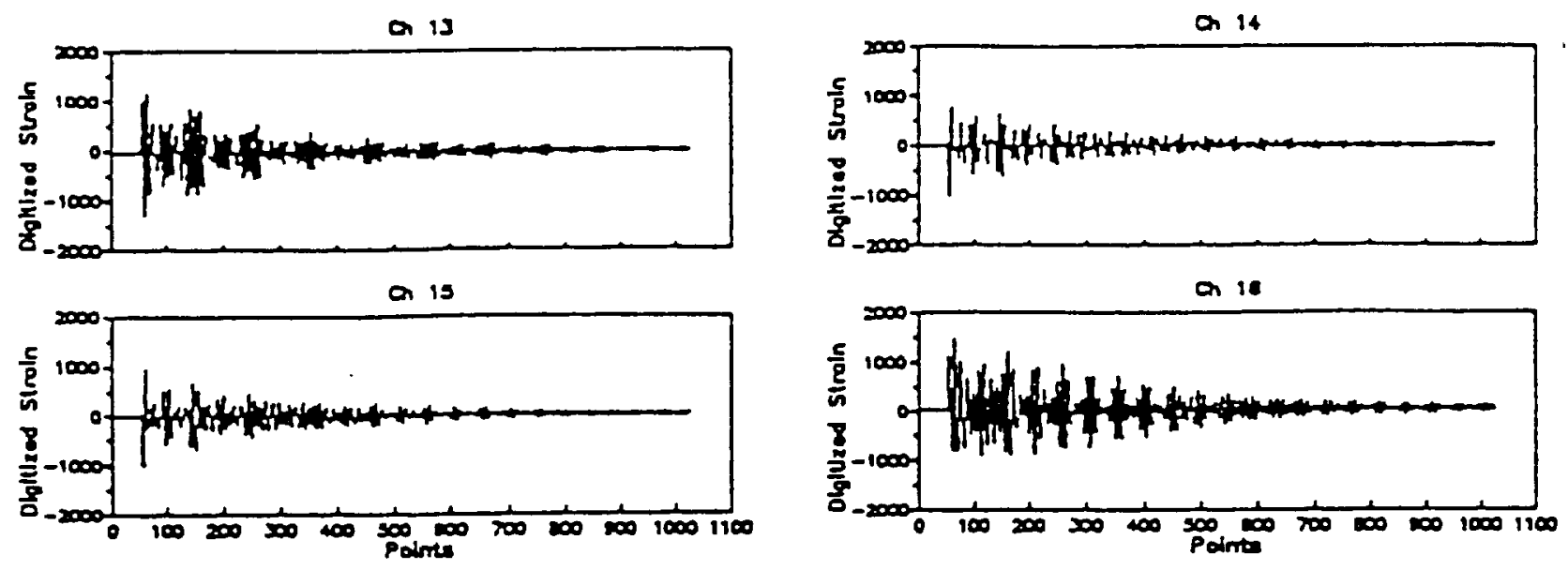

Figure 7. Typical Vibration Wave Forms
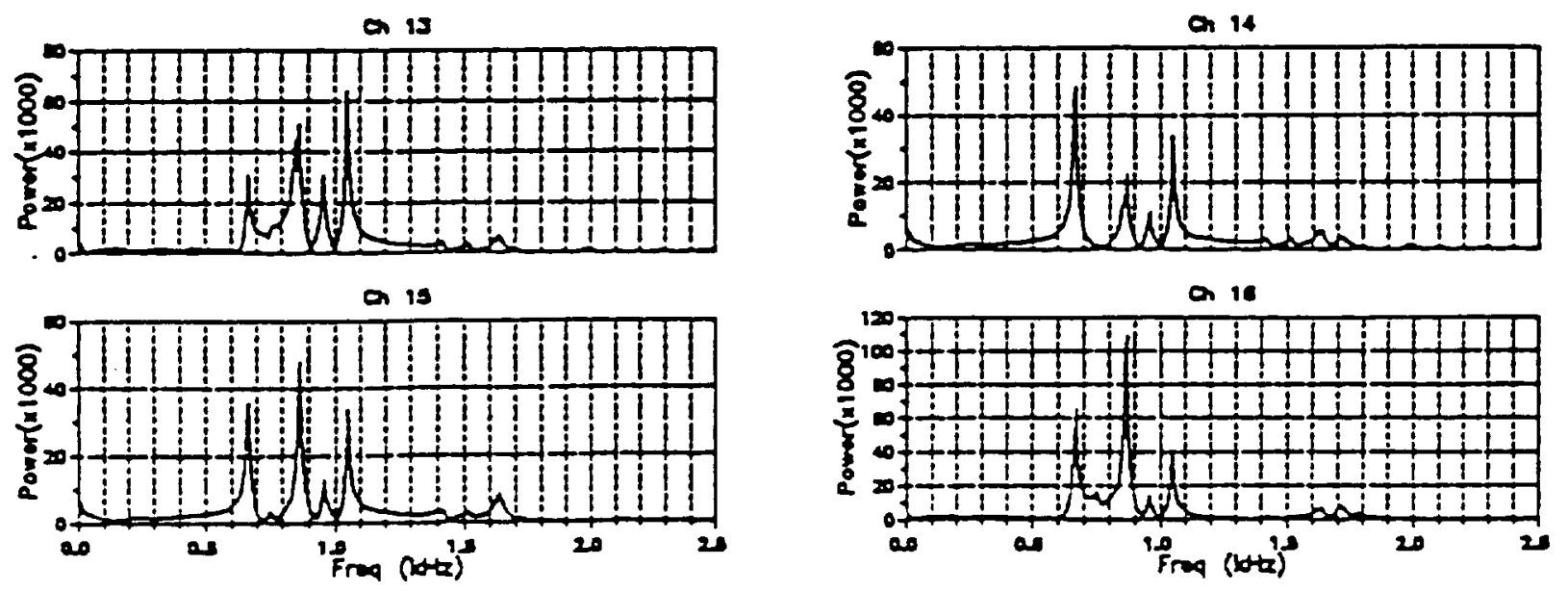

Figure 8. Corresponding Frequency Spectra 


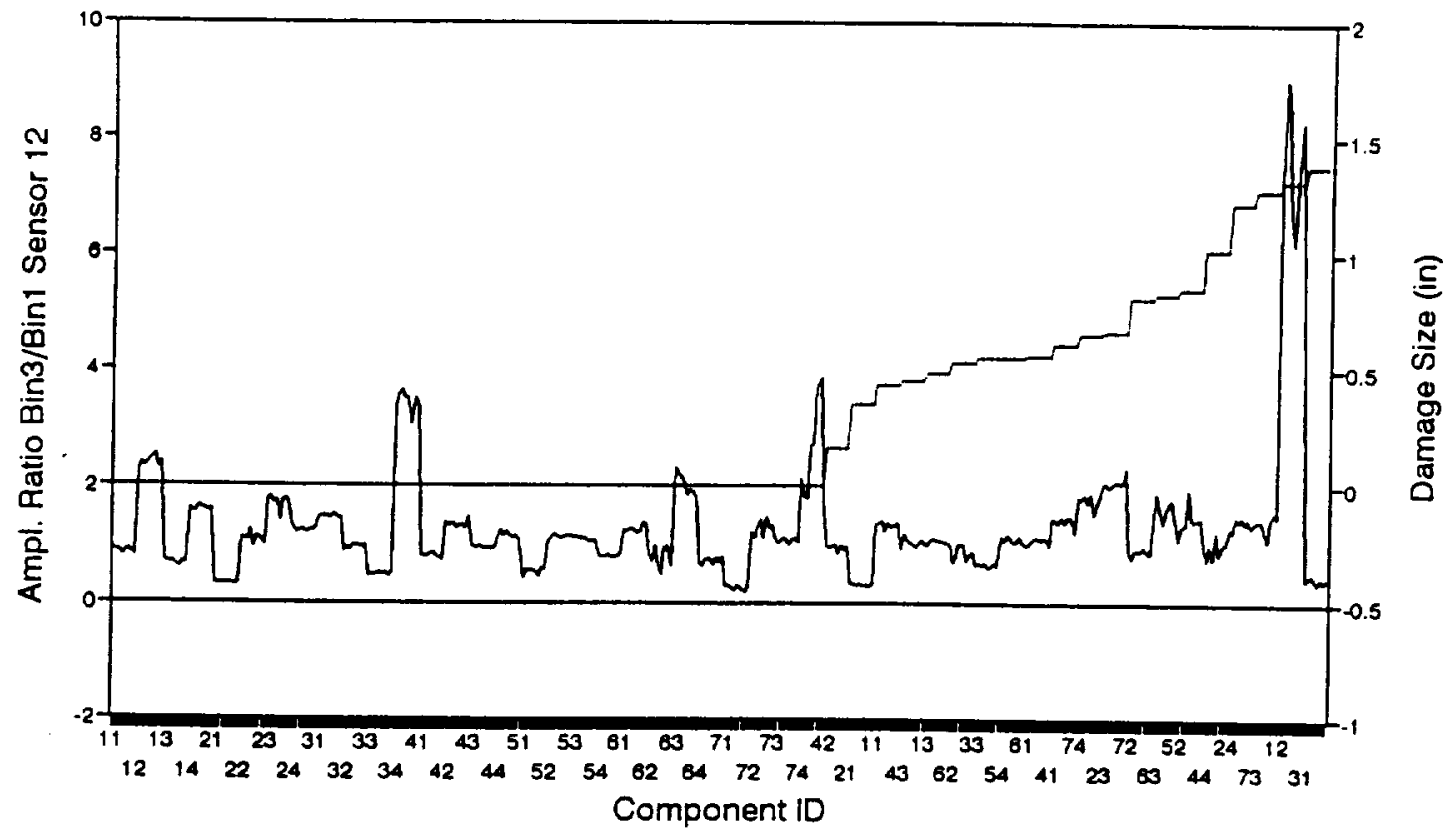

Feature Value - Damage Size

Figure 9. Comparison of Feature Without Normalization

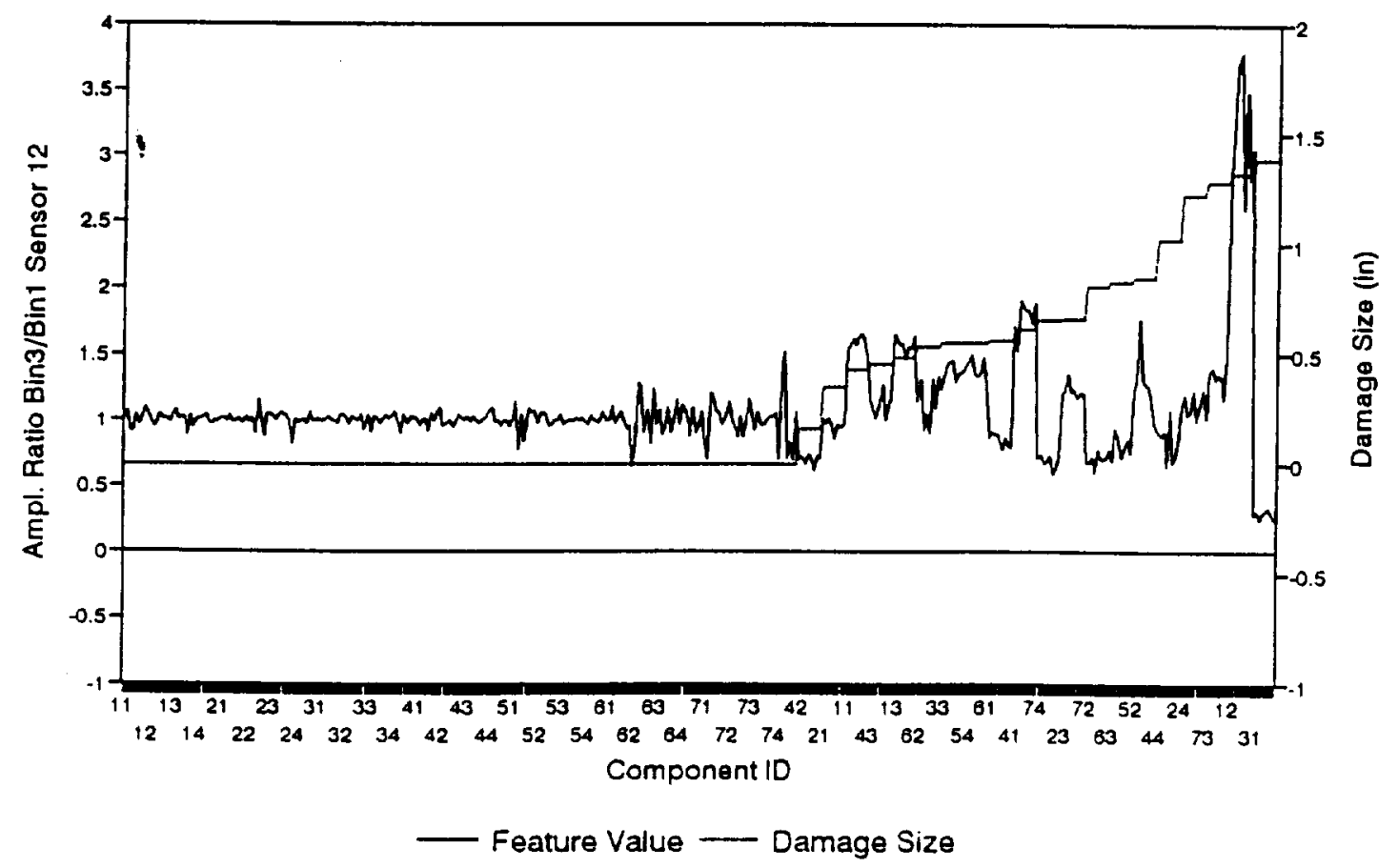

Figure 10. Comparison of Feature with Normalization 


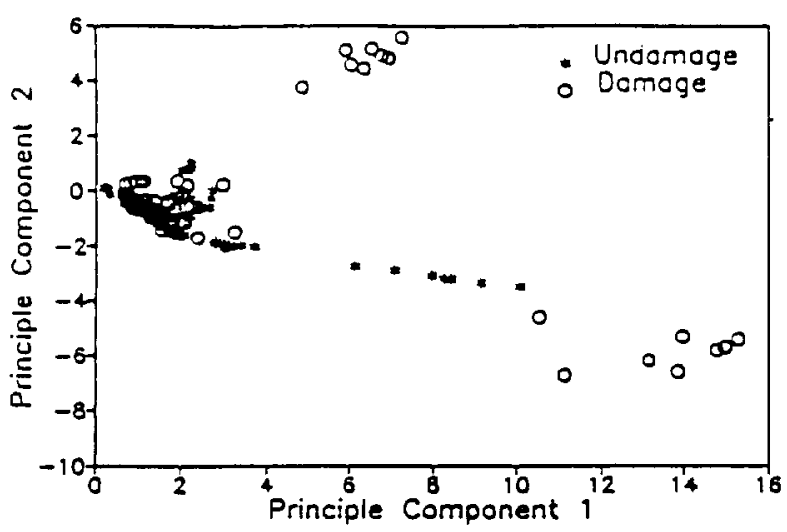

Figure 11. Distribution of Undamaged and Damaged Classes in Feature Space Using Principle Component Method

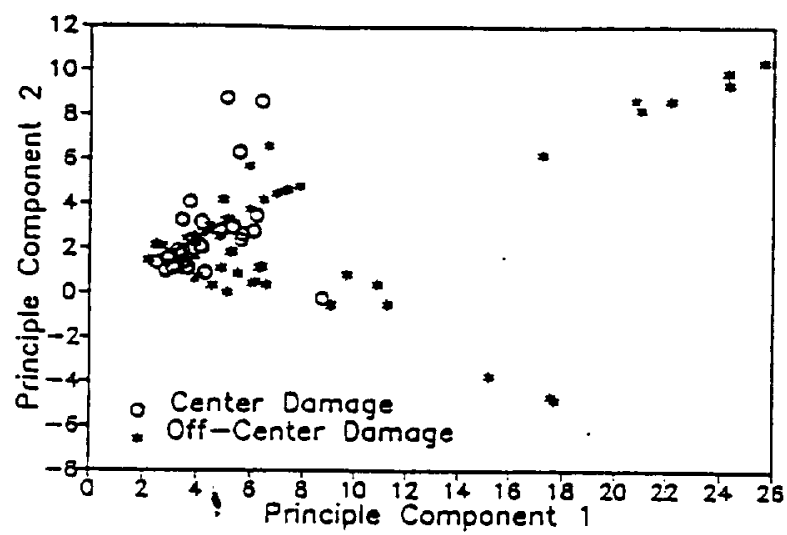

Figure 12. Distribution of Center and Off-Center Damages in Feature Space Using Principle Component Method

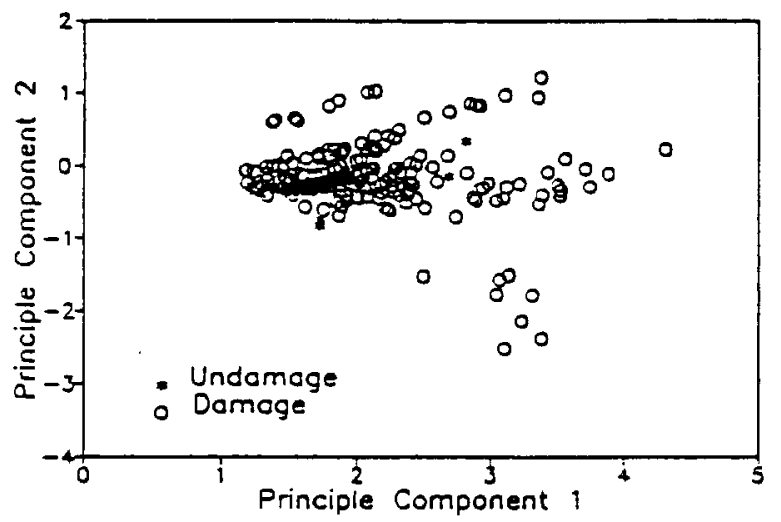

Figure 13. Distribution of Undamaged and Damaged Classes in Feature Space Using Normalized Features and Principle Component Method

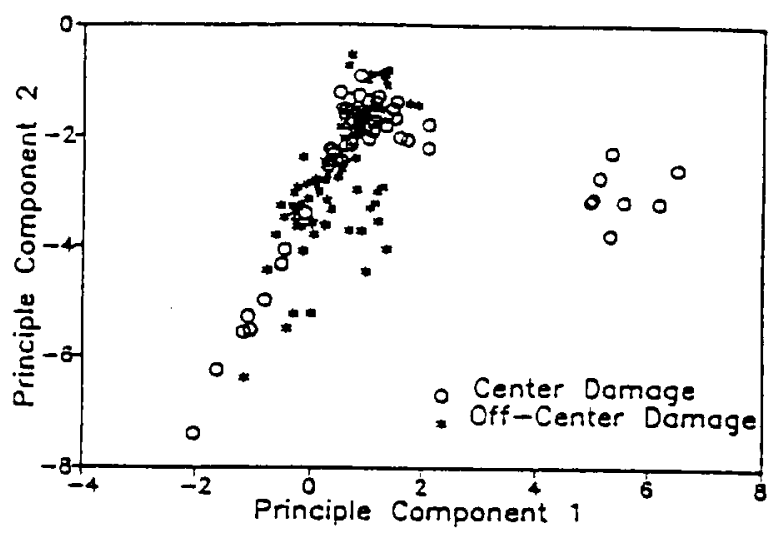

Figure 14. Distribution of Center and Off-Center Damages in Feature Space Using Normalized Features and Principle Component Method

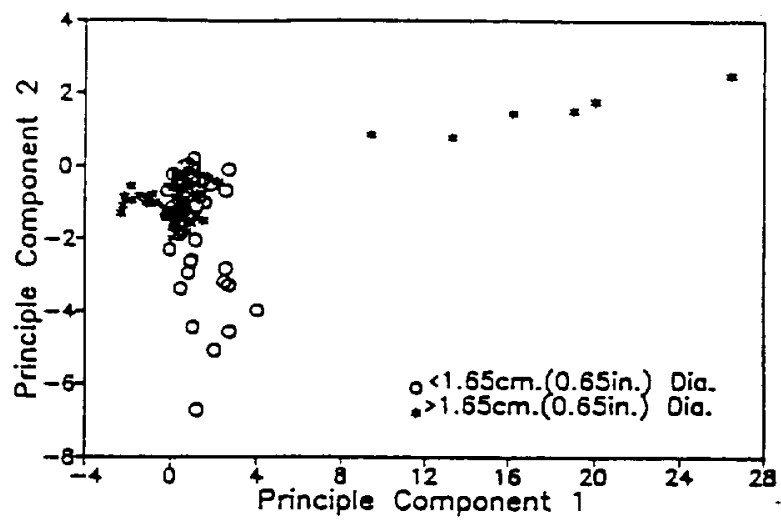

Figure 15. Distribution of Damage Size Classes in Feature Space Using Normalized Features and Principle Component Method 